\title{
Loop Mediated Isothermal Amplification for Detection of Trypanosoma brucei gambiense in Urine and Saliva Samples in Nonhuman Primate Model
}

\author{
Maina Ngotho, ${ }^{1}$ John Maina Kagira, ${ }^{2}$ Beatrice Muthoni Gachie, ${ }^{3}$ Simon Muturi Karanja, \\ Maxwell Wambua Waema, ${ }^{3}$ Dawn Nyawira Maranga, ${ }^{1}$ and Naomi Wangari Maina ${ }^{3}$ \\ ${ }^{1}$ Animal Science Department, Institute of Primate Research (IPR), P.O. Box 24481, Karen, Nairobi 00502, Kenya \\ ${ }^{2}$ Animal Health and Production Department, College of Agriculture and Natural Resources, Jomo Kenyatta University of \\ Agriculture and Technology (JKUAT), P.O. Box 62000, Nairobi 00200, Kenya \\ ${ }^{3}$ Biochemistry Department, College of Health Sciences, Jomo Kenyatta University of Agriculture and Technology (JKUAT), \\ P.O. Box 62000, Nairobi 00200, Kenya \\ ${ }^{4}$ Public Health Department, College of Health Sciences, Jomo Kenyatta University of Agriculture and Technology (JKUAT), \\ P.O. Box 62000, Nairobi 00200, Kenya
}

Correspondence should be addressed to Naomi Wangari Maina; nmaina@jkuat.ac.ke

Received 9 February 2015; Revised 22 April 2015; Accepted 3 May 2015

Academic Editor: Malcolm Jones

Copyright (C) 2015 Maina Ngotho et al. This is an open access article distributed under the Creative Commons Attribution License, which permits unrestricted use, distribution, and reproduction in any medium, provided the original work is properly cited.

Human African trypanosomiasis (HAT) is a vector-borne parasitic zoonotic disease. The disease caused by Trypanosoma brucei gambiense is the most prevalent in Africa. Early diagnosis is hampered by lack of sensitive diagnostic techniques. This study explored the potential of loop mediated isothermal amplification (LAMP) and polymerase chain reaction (PCR) in the detection of T. $b$. gambiense infection in a vervet monkey HAT model. Six vervet monkeys were experimentally infected with T. b. gambiense IL3253 and monitored for 180 days after infection. Parasitaemia was scored daily. Blood, cerebrospinal fluid (CSF), saliva, and urine samples were collected weekly. PCR and LAMP were performed on serum, CSF, saliva, and urine samples. The detection by LAMP was significantly higher than that of parasitological methods and PCR in all the samples. The performance of LAMP varied between the samples and was better in serum followed by saliva and then urine samples. In the saliva samples, LAMP had 100\% detection between 21 and $77 \mathrm{dpi}$, whereas in urine the detection it was slightly lower, but there was over $80 \%$ detection between 28 and 91 dpi. However, LAMP could not detect trypanosomes in either saliva or urine after 140 and 126 dpi, respectively. The findings of this study emphasize the importance of LAMP in diagnosis of HAT using saliva and urine samples.

\section{Introduction}

Human African trypanosomiasis (HAT) is a tropical disease that is endemic in several countries in sub-Saharan Africa. Control of sleeping sickness relies on passive case detection and it is considered to be the most cost-effective when compared to active case detection [1]. Sleeping sickness caused by T. $b$. gambiense is currently responsible for over $90 \%$ of all HAT cases [1]. Screening of the population at risk is done by antibody detection with the Card Agglutination Test for Trypanosomiasis (CATT) and confirmed by parasitological methods. Serological tests have varying sensitivities and cannot decisively differentiate between active and cured cases. Furthermore, cured patients can remain CATT seropositive for up to three years due to persisting circulating antibodies, thus prohibiting the use of antibody tests for assessment of treatment success [2]. The parasitological detection techniques also have limitations. The methods are time consuming, tedious, and prone to subjectivity. In addition, low detection rates may occur since T. b. gambiense infection is characterized by low parasitemia [3]. False negatives (CATT negative) but parasitemic cases have also been reported [4]. 
These limitations imply the need for more sensitive and specific diagnosis.

The amplification of DNA has emerged as one of the diagnostic techniques used in studies of infectious diseases [5]. Species specific genes have been used to characterize trypanosomes [6]. The discovery of the T. b. gambiensespecific glycoprotein (TgsGP) gene that is specific to the T. $b$. gambiense subspecies heralded its use as a probe for diagnosis. It is the only subspecies-specific gene for T. b. gambiense and encodes a $47 \mathrm{kDa}$ VSG-like receptor protein [7]. Amplification of this gene using PCR has successfully been used in clinical samples [8]. However, challenges of the DNA extraction protocols may affect diagnosis of trypanosome infections [9] and requirements of expensive automated thermal cyclers make PCR impractical for adoption in the field [10].

Loop mediated isothermal amplification (LAMP) is performed under isothermal conditions and relies on autocycling strand displacement DNA synthesis [11]. It requires a simple heating device and is rapid and results are easily viewed by several detection formats. The autocycling reactions lead to accumulation of a large amount of the target DNA and by-products such as magnesium pyrophosphate allowing for rapid detection using varied formats. LAMP uses four to six specially designed primers recognizing six to eight regions of the target DNA sequence resulting in a high specificity. It has been used in detection of the Trypanozoon subgenus [12], T. b. rhodesiense [13], and recently Group 1 T. b. gambiense [14]. The test has high sensitivity and specificity and does not require specialized equipment, and this makes it a suitable diagnostic test in resource poor settings and would therefore be ideal diagnosis of neglected diseases such as HAT.

The importance of experimental animal models includes controlled conditions and planned sampling among other. The vervet monkey (Chlorocebus aethiops) has been developed as a model for early stage HAT caused by T. b. gambiense [15]. Using this animal model, the performance of LAMP based on the TgsGP gene was assessed in detection of T. $b$ gambiense in serum, CSF, saliva, and urine.

\section{Materials and Methods}

2.1. Trypanosomes. Trypanosoma b. gambiense isolate IL3253 was used in this study. It was isolated from a human HAT patient from Sudan in 1982. The isolate was cryopreserved in liquid nitrogen and for infective purposes the parasites were subinoculated into immunosuppressed donor Swiss mice. At peak parasitemia, heart blood was obtained by cardiac puncture and parasites harvested and diluted to $10^{5} / \mathrm{mL}$ using phosphate saline glucose.

2.2. Experimental Animals. Six adult vervet monkeys of both sexes weighing between 2.0 and $5.0 \mathrm{~kg}$ were used in this study. They were trapped from the wild in an area known to be nonendemic for human trypanosomiasis. The animals underwent a 90-day quarantine during which they were screened for zoonotic diseases and treated for ecto- and endoparasites. They were also trained for ease of adaptation and maintained on commercial pellets (Unga Feeds
Ltd., Nairobi, Kenya) supplemented with fresh fruits and vegetables. Drinking water was provided ad libitum. The monkeys were housed in stainless steel cages at ambient room temperatures of $18-25^{\circ} \mathrm{C}$, under biosafety level II animal holding conditions. At the end of the experiment period, the animals were euthanized by injection with Euthatal (20\% sodium pentobarbitone, Rotexmedica ${ }^{\circledR}$, Trittau, Germany) via the femoral vein.

2.3. Study Design. Six monkeys were infected intravenously with approximately $10^{5}$ trypanosomes in $1 \mathrm{~mL}$ of phosphate saline glucose. The infected monkeys were monitored for a total period of 180 days after experimental infection. Parasitaemia was estimated daily using methods previously described using the rapid matching method [16] and haematocrit centrifuge technique [17].

2.4. Sample Collection. The monkeys were anaesthetized on weekly basis with ketamine hydrochloride (Rotexmedica, Trittau, Germany) at a dosage of $10 \mathrm{mg} / \mathrm{kg}$ body weight for sample collection. The samples were collected before and after infection on a weekly basis. Three $\mathrm{mL}$ of blood from the femoral artery and $1.5 \mathrm{~mL}$ of cerebrospinal fluid (CSF) via lumbar puncture were collected. Saliva samples were obtained by placing swabs under the animals tongue for ten minutes to allow for adequate wetting. Thereafter the swabs were placed in dry cryovial tubes. The urine was obtained via a collection apparatus placed on the bottom of the monkey cage and stored in $50 \mathrm{~mL}$ falcon tubes. This was done in the early morning prior to sedation. All samples were collected and stored at $-20^{\circ} \mathrm{C}$.

2.5. DNA Extraction. DNA was extracted from serum, urine, CSF, and saliva samples using genomic DNA isolation kits (Zymo Research, USA) as per manufacturer's instructions.

2.6. PCR. Amplification of T. b. gambiense-specific glycoprotein $(\mathrm{Tgs} G P)$ gene was done using primer sequences as previously described [18]. The PCR reactions (nested) were performed as described [8] using $1 \mu \mathrm{L}$ of extracted DNA in a $25 \mu \mathrm{L}$ reaction mixture. The PCR amplification was performed by incubating the samples for $15 \mathrm{~min}$ at $95^{\circ} \mathrm{C}$ followed by 45 cycles of $1 \mathrm{~min}$ at $94^{\circ} \mathrm{C}, 1 \mathrm{~min}$ at $63^{\circ} \mathrm{C}$, and $1 \mathrm{~min}$ at $72^{\circ} \mathrm{C}$ and a final extension at $72^{\circ} \mathrm{C}$ for $10 \mathrm{~min}$. Thereafter, amplified products were analyzed by electrophoresis in $2 \%$ agarose gels. Gels were stained with ethidium bromide $(0.5 \mu \mathrm{g} / \mathrm{mL})$ (Sigma, USA) and viewed under UV illumination. The negative controls: purified DNA from T. b. brucei GUTAT1 and T. $b$. rhodesiense IPR001 and distilled water. The positive control was purified T. b. gambiense IL3253 DNA.

2.7. LAMP. The TgsGP primers as previously described were used [14]. The reaction mixture of $25 \mu \mathrm{L}$ consisted of $40 \mathrm{pmol}$ of the inner primers, $5 \mathrm{pmol}$ of the outer primers, $20 \mathrm{pmol}$ of the loop primers (Inqaba biotec, SA), $0.8 \mathrm{M}$ betaine (Sigma-Aldrich, St. Louis, MO, USA), $2.8 \mathrm{mM}$ dNTPs mix, 1x Thermopol buffer (20 mM Tris- $\mathrm{HCl}$ pH 8.8, $10 \mathrm{mM}$ $\mathrm{KCl}, 2 \mathrm{mM} \mathrm{MgSO}_{4}, 10 \mathrm{mM}\left(\mathrm{NH}_{4}\right)_{2} \mathrm{SO}_{4}, 0.1 \%$ Triton X-100) 


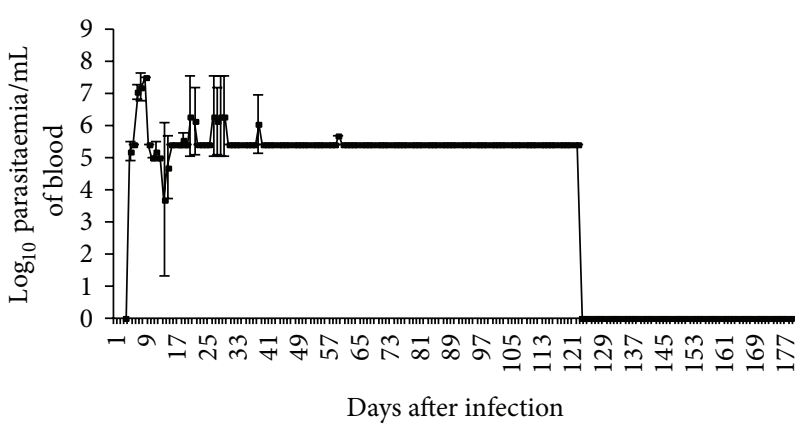

FIGURE 1: Mean daily parasitaemia of monkeys infected with T. $b$. gambiense IL3253. CSF: there was no CSF parasitosis observed during the experimental period.

(New England Biolabs, UK), and additional $4 \mathrm{mM} \mathrm{MgSO}_{4}$, 8-unit Bst DNA polymerase (New England Biolabs, UK), double distilled water, and $2 \mu \mathrm{L}$ of the template DNA. The positive and negative controls were similar to those used in the PCR reaction. The reactions were carried out in triplicate for 80 minutes in a Loopamp real-time turbidimeter LA320C (Eiken Chemical Co., Japan). Increase in turbidity indicates DNA amplification. After the reaction 1/20 dilution of SYBR green I dye (Sigma-Aldrich, St. Louis, MO, USA) was added to confirm the amplification.

2.8. Data Analysis. The percentage detection of the different tests and sample was determined and significant differences between the tests calculated using the chi-square $\left(\chi^{2}\right)$ test were determined. The differences were considered statistically significant when $p<0.05$. The agreement between tests was quantified using Cohen's kappa statistic $(k)$. Epicalc of EpiInfo 7 was used.

2.9. Ethics. All protocols and procedures used in this study were reviewed and approved by the Institute of Primate Research (IPR) Institutional Review Committee which incorporates Animal Care and Use Committee (IACUC) review (IRC/19/10).

\section{Results}

3.1. Parasitological Methods. The prepatent period was two to three days. The parasitaemia rose to a peak of $10^{7}$ trypanosomes/mL of blood between 8 and 9 days after infection (dpi). Thereafter, the parasitaemia declined and was characterized by fluctuations to a minimum $2.5 \times 10^{5}$ trypanosomes $/ \mathrm{mL}$ of blood by 123 dpi. However from 123 to $180 \mathrm{dpi}$ the parasitaemia dropped to undetectable levels (Figure 1).

3.2. PCR. The positive control (T. b. gambiense) gave the expected 308-base-pair (bp) band. The negative controls ( $T$. b. brucei and T. b. rhodesiense) were negative (Figure 2).

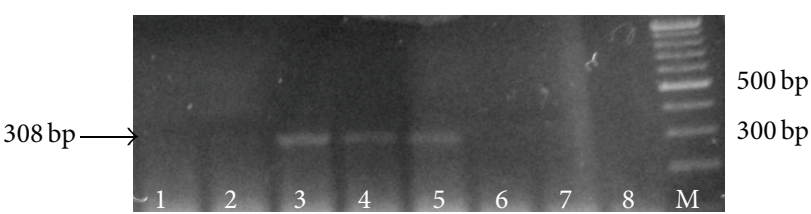

FIGURE 2: PCR results on gels after electrophoresis. Lane $1(T b b)$; Lane 2 (Tbr); Lane 3 (positive control Tbg); Lane 4 (saliva sample obtained on $14 \mathrm{dpi}$ ); Lane 5 (saliva sample obtained $28 \mathrm{dpi}$ ); Lane 6 (saliva sample obtained $56 \mathrm{dpi}$ ), Lane 7 (saliva sample obtained $70 \mathrm{dpi}$ ), Lane 8 (saliva sample obtained $84 \mathrm{dpi}$ ), and M (100 bp molecular marker).

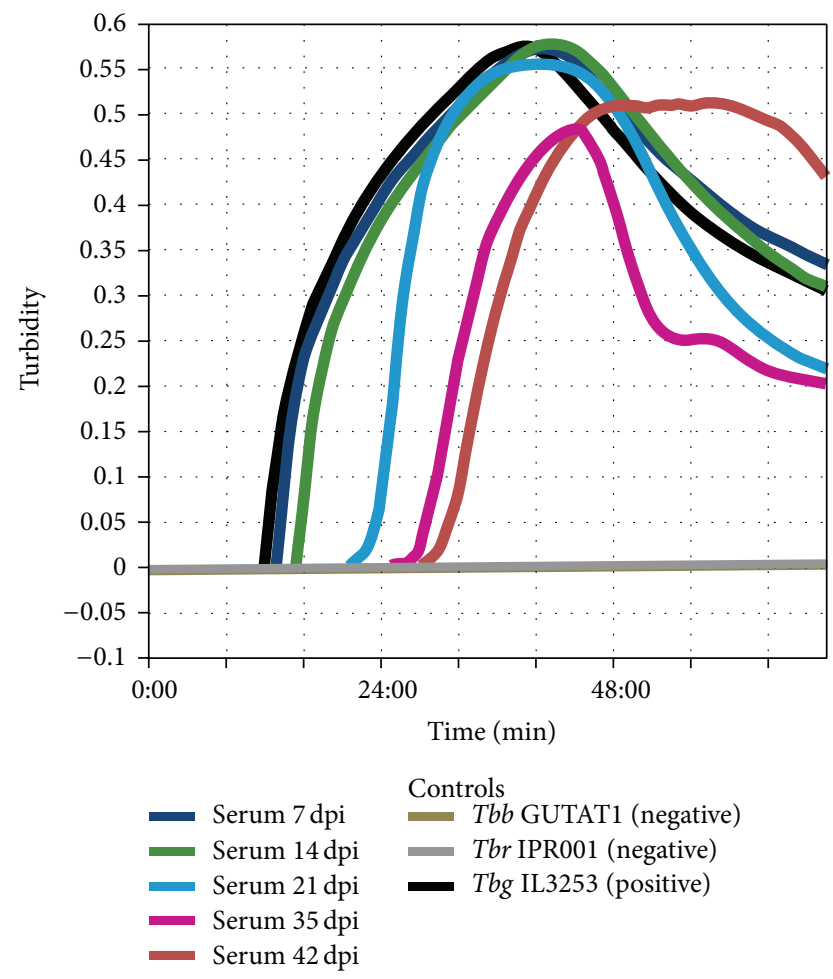

FIGURE 3: Amplification curves after LAMP reaction in turbidimeter.

3.3. LAMP. Increase in turbidity was noted for the positive control and some samples within 48 minutes of incubation. There was no increase in turbidity in the negative controls as expected (Figure 3). After addition of SYBR green I dye the positive LAMP reactions turned green while the negative ones remained orange (Figure 4).

3.4. Comparison between LAMP, PCR, and Parasitological Methods. Parasitological methods detected parasites in the infected monkeys by day 3 after infection (Figure 1). The detection rate gradually dropped and by $119 \mathrm{dpi}$ only $50 \%$ detection was obtained. Thereafter the methods could not detect the parasites. Both PCR and LAMP recorded 100\% detection in serum samples starting from $7 \mathrm{dpi}$. LAMP detected trypanosome DNA until 180 dpi but maintained $100 \%$ detection up to $133 \mathrm{dpi}$. On the other hand, PCR could 


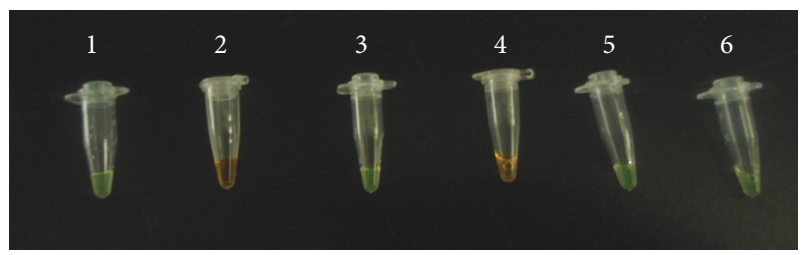

FIGURE 4: Visual appearance of LAMP results after addition of SYBR green I dye. Green color represents positive reaction while orange represents negative reaction. Tube 1: Tbg positive control, Tube 2: negative control ( $\mathrm{Tbr}$ ), Tube 3: serum sample obtained $28 \mathrm{dpi}$, Tube 4: CSF obtained $28 \mathrm{dpi}$, Tube 5: saliva sample obtained $28 \mathrm{dpi}$, and Tube 6: urine sample obtained $28 \mathrm{dpi}$.

TABLE 1: Detection (\%) of parasitological methods, PCR, and LAMP in serum, saliva, and urine determined at weekly time points in vervet monkeys infected with T. b. gambiense.

\begin{tabular}{|c|c|c|c|c|c|c|}
\hline \multirow{2}{*}{ DPI } & \multirow{2}{*}{ Parasito. } & \multicolumn{2}{|c|}{ PCR } & \multicolumn{3}{|c|}{ LAMP } \\
\hline & & Serum & Saliva & Serum & Saliva & Urine \\
\hline 7 & 100 & 100 & 17 & 100 & 33 & 0 \\
\hline 14 & 83 & 100 & 83 & 100 & 83 & 17 \\
\hline 21 & 100 & 100 & 100 & 100 & 100 & 33 \\
\hline 28 & 83 & 100 & 100 & 100 & 100 & 83 \\
\hline 35 & 67 & 100 & 100 & 100 & 100 & 100 \\
\hline 42 & 83 & 100 & 83 & 100 & 100 & 100 \\
\hline 49 & 83 & 100 & 33 & 100 & 100 & 100 \\
\hline 56 & 83 & 100 & 17 & 100 & 100 & 100 \\
\hline 63 & 83 & 100 & 17 & 100 & 100 & 83 \\
\hline 70 & 67 & 100 & 0 & 100 & 100 & 100 \\
\hline 77 & 83 & 100 & 0 & 100 & 100 & 83 \\
\hline 84 & 67 & 100 & 0 & 100 & 83 & 83 \\
\hline 91 & 50 & 83 & 0 & 100 & 83 & 83 \\
\hline 98 & 67 & 83 & 0 & 100 & 83 & 67 \\
\hline 105 & 67 & 83 & 0 & 100 & 83 & 67 \\
\hline 112 & 50 & 83 & 0 & 100 & 83 & 33 \\
\hline 119 & 50 & 67 & 0 & 100 & 33 & 17 \\
\hline 126 & 0 & 83 & 0 & 100 & 33 & 17 \\
\hline 133 & 0 & 67 & 0 & 100 & 17 & 0 \\
\hline 140 & 0 & 33 & 0 & 83 & 0 & 0 \\
\hline 147 & 0 & 33 & 0 & 83 & 0 & 0 \\
\hline 154 & 0 & 17 & 0 & 83 & 0 & 0 \\
\hline 161 & 0 & 0 & 0 & 83 & 0 & 0 \\
\hline 168 & 0 & 0 & 0 & 67 & 0 & 0 \\
\hline 175 & 0 & 0 & 0 & 67 & 0 & 0 \\
\hline 180 & 0 & 0 & 0 & 33 & 0 & 0 \\
\hline
\end{tabular}

Key: Parasito. = parasitological techniques; DPI = days after infection.

${ }^{*}$ There was no amplification in any CSF sample with either LAMP or PCR. There was also no amplification noted in urine using PCR.

only sustain the $100 \%$ detection rate up to $84 \mathrm{dpi}$. Thereafter the detection dropped and from 161-180 dpi PCR did not detect trypanosome DNA (Table 1).
TABLE 2: Duration of detection of parasites in blood, serum, saliva, urine, and CSF samples using parasitological methods, PCR, and LAMP in vervet monkeys infected with T. b. gambiense for 180 days after infection (dpi).

\begin{tabular}{lccc}
\hline Sample & Parasitology & PCR & LAMP \\
\hline Blood & $3-123$ dpi (18\%) & - & - \\
Serum & - & $7-154$ dpi (4\%) & 7-180 dpi (3\%) \\
Saliva & - & $7-63$ dpi (11\%) & $7-133$ dpi (5\%) \\
Urine & - & - & $14-126$ dpi (8\%) \\
CSF & - & - & - \\
\hline
\end{tabular}

*(1) The percentage of negative samples is given in brackets.

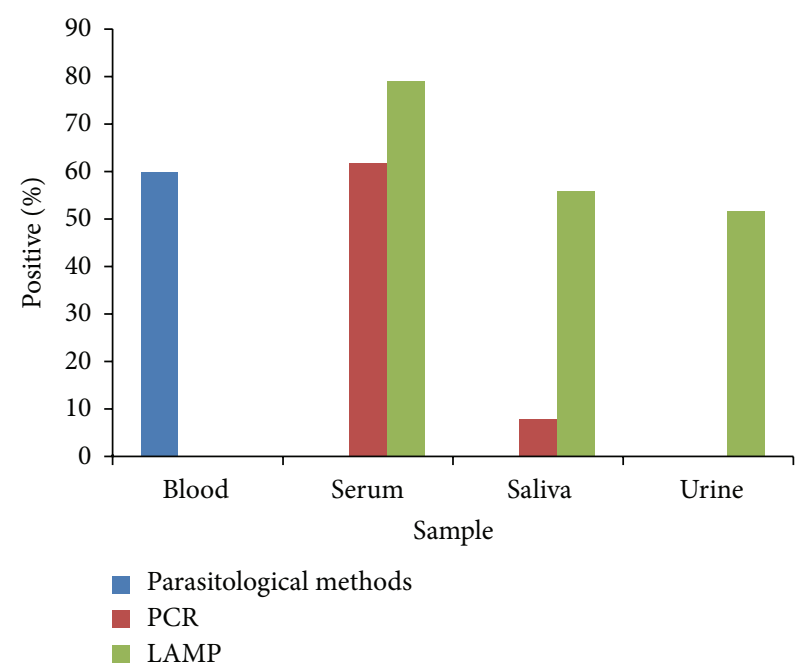

FIGURE 5: Comparison of LAMP, PCR, and parasitological methods in trypanosome mean detection rate in serum, saliva, and urine samples obtained from monkeys infected with T. b. gambiense IL3253 from 3 to 180 days after infection. There was no amplification in any CSF sample with either LAMP or PCR. There was also no amplification noted in urine using PCR.

In saliva samples, PCR detected trypanosome DNA from 7 to $63 \mathrm{dpi}$, thereafter no trypanosome DNA was detected. Between 21 and 77 dpi LAMP recorded 100\% detection in the saliva samples. The detection dropped thereafter and from 140 to 180 dpi there was no trypanosome DNA detection. LAMP detected trypanosome DNA in urine samples between days 14 and 126 after infection (Table 1). PCR did not detect trypanosome DNA in the urine samples. Neither PCR nor LAMP detected trypanosome DNA in the CSF samples. Both PCR and LAMP detected parasites beyond the period when conventional parasitological methods did. Trypanosome DNA was detected longest in serum samples followed by saliva and urine (Table 2). The detection rate of LAMP was higher than that of PCR in the serum, saliva, and urine samples (Figure 5).

There was a significant difference in detection between LAMP and PCR $(p<0.05)$ in all the samples. Similarly, the difference in detection between LAMP and parasitological methods was significant $(p<0.05)$. There was good agreement between PCR and parasitological methods in detection 
TABLE 3: Comparative analysis of LAMP, PCR, and parasitological techniques in trypanosome detection in serum, saliva, and urine samples obtained from monkeys infected with T. b. gambiense IL3253.

\begin{tabular}{lcccc}
\hline Test & Kappa value & Level of agreement & $\chi^{2}$ statistic & 2.22 \\
PCR and parasitology & $0.83(0.36-0.59)$ & Very good & 26.47 & 0.136 \\
LAMP and parasitology & $0.48(0.36-0.59)$ & Marginal & 10.42 & $<0.001$ \\
LAMP and PCR (serum) & $0.59(0.47-0.72)$ & Marginal & 79.79 & 0.0012 \\
LAMP and PCR (saliva) & $0.14(0.06-0.21)$ & Poor & 6.12 & 0.0001 \\
LAMP and PCR (urine) & 0 & Poor & 0134 \\
\hline
\end{tabular}

of trypanosomes. The difference in detection between both tests was not significant $(p>0.05)$ (Table 3$)$.

\section{Discussion}

The presence of trypanosome DNA in saliva and urine samples is of great significance given the need for noninvasive samples for diagnosis of HAT. The higher sensitivity of LAMP compared to parasitological methods and PCR is also of significance in regard to the search for new diagnostic tests for sleeping sickness. In the current study the performance of LAMP and PCR was assessed in an early stage HAT model [15].

The high number of trypanosomes positive serum samples suggests that the trypanosomes were circulating in the hemolymphatic system. The lack of detection of trypanosomeDNA in the CSF samples may mean that the trypanosomes did not cross the blood-brain barrier and hence late stage disease did not occur. Infected monkeys without trypanosomes in CSF and having WBC counts of less than 5 cells $/ \mathrm{mm}^{3}$ are regarded as being in early stage of the disease.

PCR performed better than the parasitological methods in monitoring the presence of the infection but, however, it cannot be used as a gold standard in diagnosis of trypanosomiasis because of its challenges to implement in clinical settings. The test was able to detect trypanosome infection for a longer duration compared to the parasitological methods which are regarded as the gold standard. PCR detected trypanosome DNA in the saliva samples during early infection corresponding to the period of high parasitaemia. The lack of detection in urine samples could be due to the presence of inhibitors such as urea and uric acid [19] and elevated acidic conditions that may inactivate the highly sensitive Taq DNA polymerase enzyme used in PCR reactions. This enzyme used is also easily inactivated by tissue and blood derived inhibitors [20-22]. Similarly, in a previous study on $T$. $b$. rhodesiense, trypanosome DNA was not detected in urine samples of infected vervet monkeys [unpublished data]. In this study, all the CSF samples were negative for trypanosome DNA although previous studies have shown that PCR on CSF samples has high sensitivity in staging of HAT [23]. Optimization of reaction conditions is a major setback in development of PCR as a diagnostic tool for HAT. This has especially been noted in samples from serologically positive but aparasitemic patients [9]. In addition, PCR is cost restrictive due to the need for specialized equipment such as automated thermal cyclers and the presence of cold chain to preserve reagents. Thus, recent studies have focused on development of tests such as LAMP which can overcome some of these challenges $[10,24]$.

LAMP performed better than PCR and parasitological methods as demonstrated by its higher detection rate. In contrast to PCR, LAMP detected trypanosome DNA in urine samples in this study. Indeed, the test detected trypanosome DNA during periods of low parasitaemia when both PCR and parasitology were negative. The significant difference in detection proves that the performance of LAMP was markedly better than that of both parasitological methods and PCR. This is possibly due to the use of Bst polymerase that unlike Taq polymerase is hardly inhibited by impurities [25]. The detection of trypanosomal DNA in urine and saliva samples is of great value because they are noninvasive samples and are an improvement from the current blood and CSF samples [26].

Trypanosomes have been found to be present in many organs and body fluids. There has been demonstrated localization of T. $b$. brucei in kidney glomeruli of infected rats [27] and T. lewisi in kidney capillaries of infected rats [28]. Filtration of the parasite or its DNA may explain the presence of trypanosomal DNA in the urine of the infected monkeys used in this study. However, formation of ammonia in exposed urine causes degradation of DNA and may lower the sensitivity of the tests.

Trypanosome DNA was also detected in the saliva samples of the infected monkeys. It is possible that parasites could have seeped into salivary ducts from either the blood or lymphatic systems. Saliva has a higher $\mathrm{pH}$ as compared to urine hence reducing the likelihood of DNA deterioration.

In this study, we targeted TgsGP gene a single gene which encodes a protein specific to the T. b. gambiense subspecies and hence ideal for specific detection of T. b. gambiense [29]. The LAMP detection rate varied and was $100 \%$ in some durations depending on the sample used. The sensitivity appeared to be affected by parasitaemia and hence the amount of DNA in the sample. The concentration of the trypanosomal DNA in the sample was however not assessed. The highest mean detection rate $(78.9 \%)$ over the entire 180 days experimental period was lower than obtained using a repetitive element (repetitive insertion mobile element, RIME) [30]. A repetitive DNA like RIME means that many copies of DNA are available for amplification and hence greater sensitivity is expected. However, it is important to note that in that study $90 \%$ sensitivity was obtained with the samples from parasitological confirmed patients. We recommend that the sensitivity of 
LAMP targeting the TgsGP gene be further evaluated in clinical samples and especially the noninvasive samples such as urine and saliva. There is also promising development in serological tests. A number of rapid tests are under clinical evaluation in many countries [31]. Combined use of the rapid serological tests and molecular techniques such as LAMP will enhance early diagnosis of HAT in the rural Africa where the disease is endemic.

\section{Conflict of Interests}

The authors declare that they have no conflict of interests.

\section{Acknowledgments}

This work was funded by the Institute of Primate Research (IPR) and JKUAT-RPE program. The authors are grateful to the technical assistance provided by Tom Adino and Samson Mutura of IPR.

\section{References}

[1] J. R. Franco, P. P. Simarro, A. Diarra, J. A. Ruiz-Postigo, and J. G. Jannin, "The journey towards elimination of gambiense human African trypanosomiasis: not far, nor easy," Parasitology, vol. 141, no. 6, pp. 748-760, 2014.

[2] V. Lejon, D. M. Ngoyi, M. Boelaert, and P. Büscher, "A CATT negative result after treatment for human african trypanosomiasis is no indication for cure," PLoS Neglected Tropical Diseases, vol. 4, no. 1, article e590, 2010.

[3] W. O. Inojosa, I. Augusto, Z. Bisoffi et al., "Diagnosing human African trypanosomiasis in Angola using a card agglutination test: observational study of active and passive case finding strategies," The British Medical Journal, vol. 332, no. 7556, pp. 1479-1481, 2006.

[4] A. Garcia, V. Jamonneau, E. Magnus et al., "Follow-up of card agglutination trypanosomiasis test (CATT) positive but apparently aparasitaemic individuals in Côte d'Ivoire: evidence for a complex and heterogeneous population," Tropical Medicine and International Health, vol. 5, no. 11, pp. 786-793, 2000.

[5] A. García-Quintanilla, J. González-Martín, G. Tudó, M. Espasa, and M. T. J. de Anta, "Simultaneous identification of Mycobacterium genus and Mycobacterium tuberculosis complex in clinical samples by 5/-exonuclease fluorogenic PCR," Journal of Clinical Microbiology, vol. 40, no. 12, pp. 4646-4651, 2002.

[6] I. Malele, L. Craske, C. Knight et al., "The use of specific and generic primers to identify trypanosome infections of wild tsetse flies in Tanzania by PCR," Infection, Genetics and Evolution, vol. 3, no. 4, pp. 271-279, 2003.

[7] M. Berberof, D. Pérez-Morga, and E. Pays, "A receptor-like flagellar pocket glycoprotein specific to Trypanosoma brucei gambiense," Molecular and Biochemical Parasitology, vol. 113, no. 1, pp. 127-138, 2001.

[8] N. Maina, K. J. Maina, P. Mäser, and R. Brun, "Genotypic and phenotypic characterization of Trypanosoma brucei gambiense isolates from Ibba, South Sudan, an area of high melarsoprol treatment failure rate," Acta Tropica, vol. 104, no. 2-3, pp. 8490, 2007.

[9] P. Solano, V. Jamonneau, P. N’Guessan et al., “Comparison of different DNA preparation protocols for PCR diagnosis of
Human African Trypanosomosis in Côte d'Ivoire," Acta Tropica, vol. 82, no. 3, pp. 349-356, 2002.

[10] N. Kuboki, N. Inoue, T. Sakurai et al., "Loop-mediated isothermal amplification for detection of African trypanosomes," Journal of Clinical Microbiology, vol. 41, no. 12, pp. 5517-5524, 2003.

[11] T. Notomi, H. Okayama, H. Masubuchi et al., "Loop-mediated isothermal amplification of DNA," Nucleic Acids Research, vol. 28, no. 12, p. e63, 2000.

[12] Z. K. Njiru, A. S. J. Mikosza, E. Matovu et al., "African trypanosomiasis: sensitive and rapid detection of the subgenus Trypanozoon by loop-mediated isothermal amplification (LAMP) of parasite DNA," International Journal for Parasitology, vol. 38, no. 5, pp. 589-599, 2008.

[13] Z. K. Njiru, A. S. J. Mikosza, T. Armstrong, J. C. Enyaru, J. M. Ndung'u, and A. R. C. Thompson, "Loop-mediated isothermal amplification (LAMP) method for rapid detection of Trypanosoma brucei rhodesiense," PLoS Neglected Tropical Diseases, vol. 2, no. 1, article e147, 2008.

[14] Z. K. Njiru, R. Traub, J. O. Ouma, J. C. Enyaru, and E. Matovu, "Detection of group 1 Trypanosoma brucei gambiense by loop-mediated isothermal amplification," Journal of Clinical Microbiology, vol. 49, no. 4, pp. 1530-1536, 2011.

[15] B. M. Gachie, J. M. Kagira, S. M. Karanja, M. W. Waema, J. M. Ngotho, and N. W. N. Maina, "Trypanosoma brucei gambiense infection in vervet monkeys: a potential model for early-stage disease," Journal of Medical Primatology, vol. 43, no. 2, pp. 7277, 2014.

[16] W. J. Herbert and W. H. Lumsden, "Trypanosoma brucei: a rapid 'matching' method for estimating the host's parasitem," Experimental Parasitology, vol. 40, no. 3, pp. 427-431, 1976.

[17] P. T. Woo, "The haematocrit centrifuge technique for the diagnosis of African trypanosomiasis.," Acta Tropica, vol. 27, no. 4, pp. 384-386, 1970.

[18] M. Radwanska, F. Claes, S. Magez et al., "Novel primer sequences for polymerase chain reaction-based detection of Trypanosoma brucei gambiense," The American Journal of Tropical Medicine and Hygiene, vol. 67, no. 3, pp. 289-295, 2002.

[19] G. Khan, H. O. Kangro, P. J. Coates, and R. B. Heath, "Inhibitory effects of urine on the polymerase chain reaction for Cytomegalovirus DNA," Journal of Clinical Pathology, vol. 44 , no. 5, pp. 360-365, 1991.

[20] A. Akane, K. Matsubara, H. Nakamura, S. Takahashi, and K. Kimura, "Identification of the heme compound co-purified with deoxyribonucleic acid (DNA) from bloodstains, a major inhibitor of polymerase chain reaction (PCR) amplification," Journal of Forensic Sciences, vol. 39, no. 2, pp. 362-372, 1994.

[21] W. A. Al-Soud, L. J. Jönsson, and P. Rådström, "Identification and characterization of immunoglobulin $\mathrm{G}$ in blood as a major inhibitor of diagnostic PCR," Journal of Clinical Microbiology, vol. 38, no. 1, pp. 345-350, 2000.

[22] L. Bélec, J. Authier, M.-C. Eliezer-Vanerot, C. Piédouillet, A. S. Mohamed, and R. K. Gherardi, "Myoglobin as a polymerase chain reaction ( $\mathrm{pcr}$ ) inhibitor: a limitation for pcr from skeletal muscle tissue avoided by the use of Thermus thermophilus polymerase," Muscle and Nerve, vol. 21, no. 8, pp. 1064-1067, 1998.

[23] S. Deborggraeve, V. Lejon, R. A. Ekangu et al., "Diagnostic accuracy of pcr in gambiense sleeping sickness diagnosis, staging and post-treatment follow-up: a 2-year longitudinal study," PLoS Neglected Tropical Diseases, vol. 5, no. 2, article e972, 2011. 
[24] Z. K. Njiru, "Rapid and sensitive detection of human African Trypanosomiasis by loop-mediated isothermal amplification combined with a lateral-flow dipstick," Diagnostic Microbiology and Infectious Disease, vol. 69, no. 2, pp. 205-209, 2011.

[25] O. M. M. Thekisoe, N. Kuboki, A. Nambota et al., "Speciesspecific loop-mediated isothermal amplification (LAMP) for diagnosis of trypanosomosis," Acta Tropica, vol. 102, no. 3, pp. 182-189, 2007.

[26] WHO/TDR, Report of the Scientific Group Meeting on African Trypanosomiasis, WHO/TDR, Seneva, Switzerland, 2001.

[27] J. Simaren and M. Ogunnaike, "Urinary biochemical changes, histopathologic effect of kidney damage observed in rats infected with Trypanosoma b. brucei," Tropical Medicine and Parasitology, vol. 11, pp. 35-46, 1989.

[28] J. O. Simaren, "Ultrastructural demonstration and glomerular cytopathology associated with Trypanosoma lewisi," in Proceedings of the 3rd International Congress of Parasitology, vol. 3, pp. 215-233, Springer, Paris, France, 1974.

[29] W. Gibson, L. Nemetschke, and J. Ndung'u, "Conserved sequence of the TgsGP gene in Group 1 Trypanosoma brucei gambiense," Infection, Genetics and Evolution, vol. 10, no. 4, pp. 453-458, 2010.

[30] P. Mitashi, E. Hasker, D. M. Ngoyi et al., "Diagnostic accuracy of loopamp Trypanosoma brucei detection kit for diagnosis of human African trypanosomiasis in clinical samples," PLoS Neglected Tropical Diseases, vol. 7, no. 10, Article ID e2504, 2013.

[31] P. Büscher, P. Mertens, T. Leclipteux et al., "Sensitivity and specificity of HAT Sero-K-SeT, a rapid diagnostic test for serodiagnosis of sleeping sickness caused by Trypanosoma brucei gambiense: a case-control study," The Lancet Global Health, vol. 2, no. 6, pp. e359-e363, 2014. 

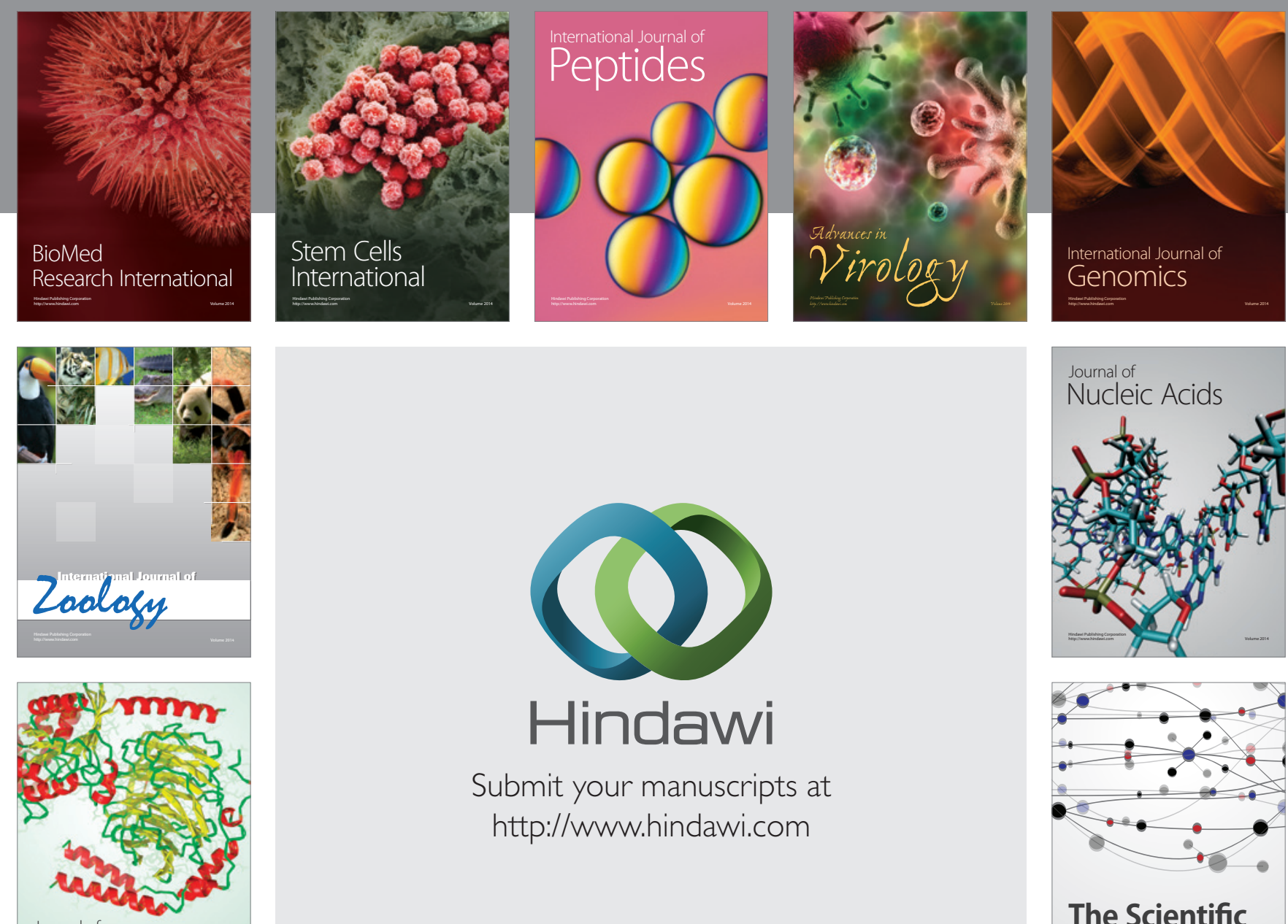

Submit your manuscripts at

http://www.hindawi.com

Journal of
Signal Transduction
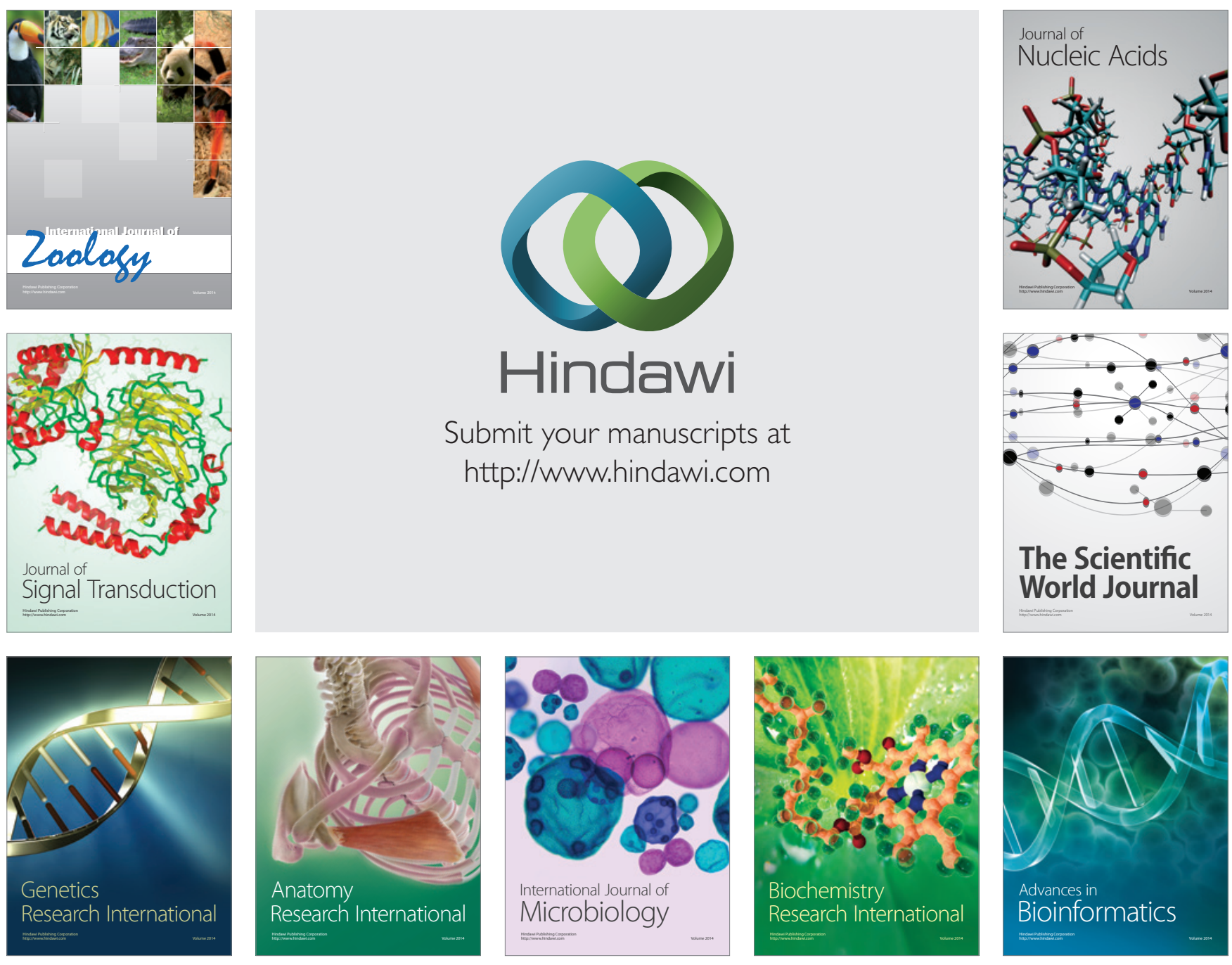

The Scientific World Journal
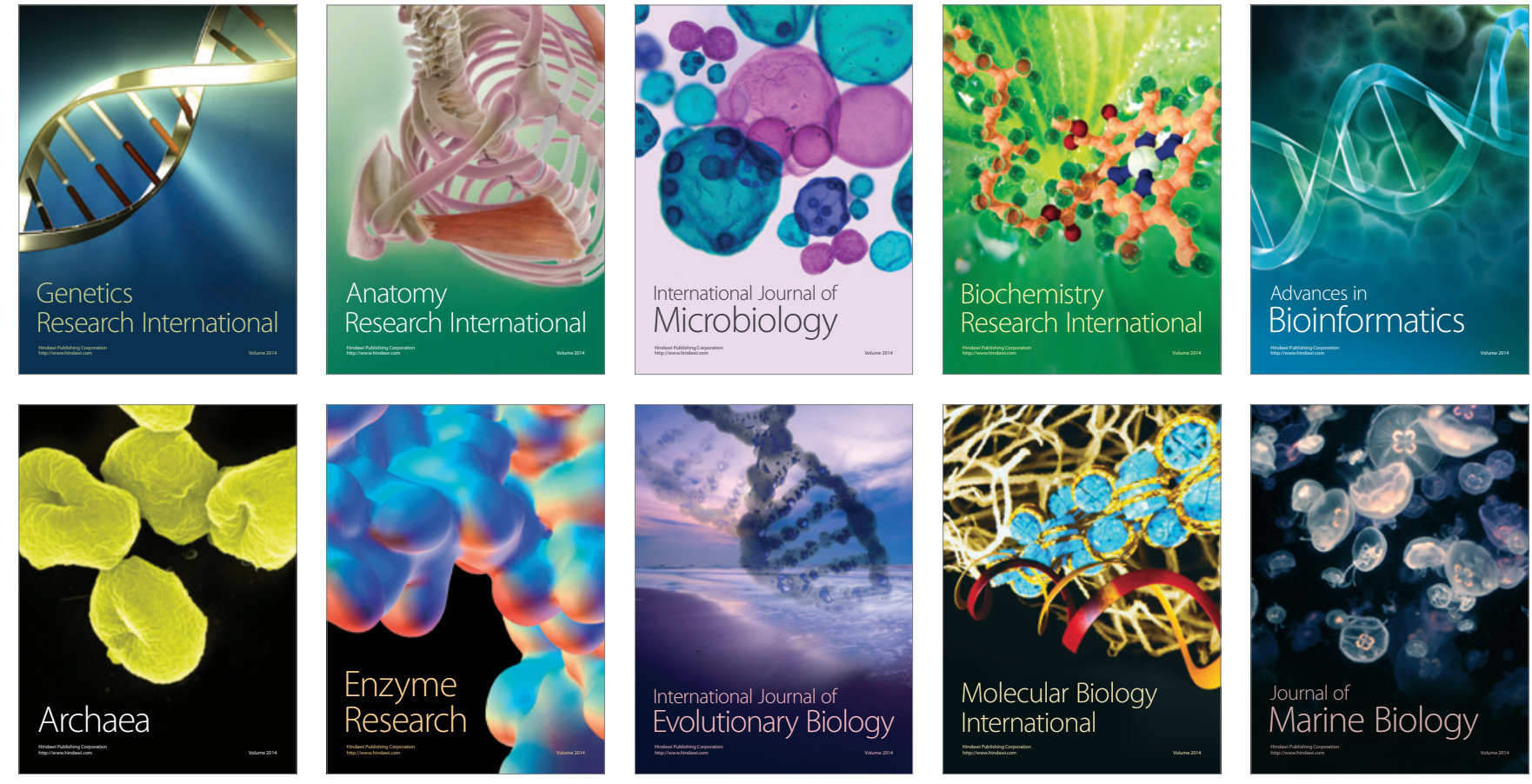